Gast-Editoren

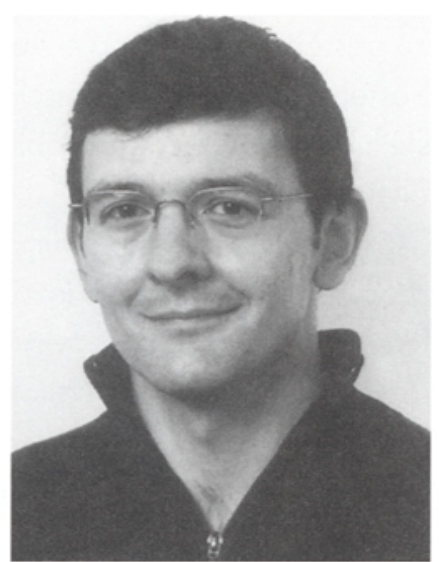

Tumasch Reichenbacher

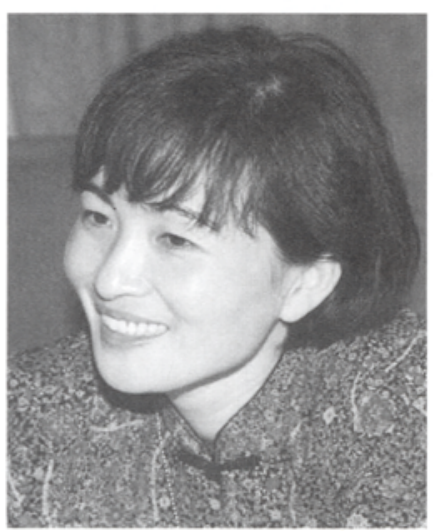

Liqiv Meng

\section{Mobile Kartographie}

Gern haben wir die Anregung des bisherigen Hauptschriftleiters dieser Zeitschrift, Uwe Fichtner, aufgegriffen, um Ihnen in einer kleinen Aufsatzreihe jenes neve Forschungs- und Arbeitsgebiet der Kartographie nahe zu bringen, das wir unter dem Begriff "mobile Kartographie" im KN-Heft 4/2002 IS. 164 ff.l zur Diskussion gestellt haben. Und gern sind Kolleginnen und Kollegen nicht nur in Deutschland, sondern auch im benachbarten Ausland unserer Bitte nachgekommen, entsprechende Beiträge zu verfassen - mit dem (erfreulichen) Ergebnis, dass wir Ihnen dieses hoch aktuelle kartographische Tätigkeitsfeld in insgesamt acht Aufsätzen Ivier im vorliegenden und - aus Platzgründen - weitere vier im nächsten Heft, in KN 2/2003) vorstellen können.

Zum Auftakt geben wir eine kurze Einführung in die mobile Kartographie. Anschließend diskutiert A. Zipf IHeidelbergl grundlegende Fragen der personalisierten und kontextbezogenen Kartengenerierung und leitet grundlegende Forschungsfragen ab. M. Galanda und $A$. Cecconi (Zürich) beschreiben die Möglichkeiten des adaptiven Zooming in der Internetkartographie; ihr Prototyp kombiniert vordefinierte LoDs sowie Methoden der Echtzeitgeneralisierung - ein Ansatz, der sich auch auf den Bereich der mobilen Kartographie übertragen lässt. Mit Navigationsaufgaben beschäftigen sich G. Gartner und Co-Autor/innen (Wienl, wobei sie schwerpunktartig den Einsatz multimedialer kartographischer Darstellungen in der Geokommunikation am Beispiel von Fußgängernavigationssystemen aufzeigen.
In Heft 2/2003 werden sich zunächst B. Elias und M. Seester (Hannover) mit einer Methode zur Anreicherung von Wegebeschreibungen befassen; sie gehen insbesondere auf die so wichtigen Landmarken ein und zeigen, wie diese aus bestehenden Geodaten extrahiert, generalisiert und auf mobilen Endgeräten visualisiert werden können. In einem weiteren Beitrag stellt W. Lopau (Houdan/F) einen Lösungsansatz für die automatische räumliche Orientierung von Karten auf mobilen Endgeräten vor. D. Burghardt und CoAutoren (Zürich) vermitteln einen Uberblick über das EU-Projekt WebPark, wobei auch sie sich mit der personalisierten und adaptierten Visualisierung von Geoinformation im Bereich von LBS beschäftigen, hier speziell für Erholungs- bzw. Naturschutzgebiete. In einem abschließenden Kurzbeitrag geben wir einen Ausblick auf die wichtigsten Forschungsfragen in der aktuellen Situation der mobilen Kartographie.

Wir hoffen, dass diesem - nach KN 5/2002 und KN 6/2002 - neverlichen Themenheft der KN in Zukunft weitere thematisch fokussierte Hefte folgen werden und bedanken uns bei dem bisherigen Hauptschriftleiter, Prof. Fichtner, sowie seinem Ikommissarischenl Nachfolger, Prof. Dodt, für die Möglichkeit, die mobile Kartographie als thematischen Schwerpunkt in den $\mathrm{KN}$ zu präsentieren. Das professionelle redaktionelle Management sowie die Freiheit und die Geduld, die uns entgegen gebracht worden sind, haben uns die Arbeit sehr erleichtert.

Tumasch Reichenbacher, Liqiu Meng TU München 